Bundesgesundheitsbl 2013 · 56:1017-1022

DOI 10.1007/s00103-013-1781-7

๑) Springer-Verlag Berlin Heidelberg 2013
Bekanntmachung des Bundesinstituts für Risikobewertung

\section{Gesundheitliche Beurteilung von Materialien und Gegenständen für den Lebensmittelkontakt im Rahmen des Lebensmittel- und Futtermittelgesetzbuches}

\author{
215. Mitteilung
}

\section{Polyamide}

Stand vom 01.06.2013

Die Empfehlung, zuletzt geändert nach dem Stand vom 01.01.2012 [Bundesgesundheitsbl 55 (2012) 291-294], wird wie folgt ergänzt:

Unter der Nr. 1 wird nach der einleitenden Formulierung („Die im Folgenden gegebene Bewertung bezieht sich auf Polymere aus den folgenden monomeren Ausgangsstoffen:") folgende Substanz ergänzt:

"Adipinsäure“

\section{Kunststoff-Dispersionen}

Stand vom 01.06.2013

Die Empfehlung, zuletzt geändert nach dem Stand vom 01.01.2012 [Bundesgesundheitsblatt - Gesundheitsforschung - Gesundheitsschutz 55 (2012) 291-294], wird wie folgt geändert und ergänzt: Der Name der Empfehlung wird wie folgt geändert: „Polymer-Dispersionen“

Die Einleitung wird einschließlich Teil A Nr. 1 wie folgt geändert:

"Gegen die Verwendung von weichmacherfreien Beschichtungen auf der Grundlage von Polymer-Dispersionen bei Bedarfsgegenständen im Sinne von $\$ 2$ Abs. 6 Nr. 1 des Lebensmittel- und Futtermittelgesetzbuches (siehe
Teil A dieser Empfehlung) sowie als Käseüberzüge, die nicht zum Verzehr bestimmt sind (siehe Teil B dieser Empfehlung), bestehen keine Bedenken, sofern sich die beschichteten Bedarfsgegenstände bzw. die Käseüberzüge für den vorgesehenen Zweck eignen und folgende Voraussetzungen erfüllt sind:

Teil A: Beschichtungen auf der Grundlage von Polymer-Dispersionen für Bedarfsgegenstände für den Lebensmittelkontakt (z. B. Klebstoffe oder Papierbeschichtungen)

\section{Als Monomere für die Polymer-Disper-} sionen dürfen verwendet werden:

Es dürfen die in der Verordnung (EU) Nr. 10/2011 aufgeführten Monomere unter Einhaltung der dort festgelegten spezifischen Grenzwerte verwendet werden.

Darüber hinaus dürfen folgende Monomere eingesetzt werden:

a) Acrylsäure- und Methacrylsäureester von Alkoholen der Kettenlänge $\mathrm{C}_{1}-$ $\mathrm{C}_{18}$, die einwertig, gesättigt und linear sind $\left(\mathrm{H}-\left(\mathrm{CH}_{2}\right)_{1-18}-\mathrm{OH}\right)$, und Etheralkoholen der Kettenlänge $\mathrm{C}_{1}-\mathrm{C}_{18}$, die einwertig, gesättigt und linear sind $\left(\mathrm{H}-\left(\mathrm{CH}_{2}\right)_{1-18}-\mathrm{O}-\left(\mathrm{CH}_{2}\right)_{1-18}-\mathrm{OH}\right)$

b) Vinylester von aliphatischen gesättigten Carbonsäuren der Kettenlänge $\mathrm{C}_{1}-\mathrm{C}_{18}$ c) Maleinsäure- und Fumarsäureester von einwertigen aliphatischen gesättigten Alkoholen der Kettenlänge $\mathrm{C}_{1}-\mathrm{C}_{18}$ oder von einwertigen aliphatischen ungesättigten Alkoholen der Kettenlänge $\mathrm{C}_{3}-\mathrm{C}_{18}$

d) Ester aliphatischer Carbonsäuren der Kettenlänge $\mathrm{C}_{3}-\mathrm{C}_{12}$ mit ungesättigten Alkoholen der Kettenlänge $\mathrm{C}_{3}-\mathrm{C}_{18}$

e) Ester von ungesättigten aliphatischen Dicarbonsäuren mit Polyethylenglykolen und/oder Polypropylenglykolen

f) Vinylether von einwertigen aliphatischen gesättigten Alkoholen der Kettenlänge $\mathrm{C}_{1}-\mathrm{C}_{18}$

g) Vinylsulfonsäure, höchstens 8\%. Sofern eine Vernetzung durch nachträgliche Behandlung sichergestellt ist, darf der Anteil höchstens 12\% betragen.

h) Halbester der Malein- bzw. Fumarsäure und der Itaconsäure mit einwertigen aliphatischen gesättigten Alkoholen der Kettenlänge $\mathrm{C}_{1}-\mathrm{C}_{18}$, höchstens $8 \%$. Sofern eine Vernetzung durch nachträgliche Behandlung sichergestellt ist, darf der Anteil

i) Acrylsäure- und Methacrylsäureester von zweiwertigen aliphatischen Alkoholen der Kettenlänge $\mathrm{C}_{2}-\mathrm{C}_{18}$

j) Divinyl- und Diallylester von gesättigten und ungesättigten aliphatischen Dicarbonsäuren der Kettenlänge $\mathrm{C}_{3}-\mathrm{C}_{18}$ höchstens $25 \%$ betragen. 
k) Acrylsäurevinylester

l) Acrylsäureallylester

m)Crotonsäurevinylester

n) Crotonsäureallylester

o) Triallylcyanurat

p) 2-Sulfoethylmethacrylat, höchstens $1,5 \%$. Beschichtungen, die unter Verwendung dieses Monomers hergestellt sind, dürfen nicht für die Verpackung von flüssigen Lebensmitteln verwendet werden. Die in der Verordnung (EU) Nr. 10/2011 festgelegte Begrenzung ist zu beachten.

Schließen die oben aufgeführten generischen Begriffe Einzelsubstanzen ein, welche in der Verordnung (EU) Nr. 10/2011 geregelt sind, so sind die dort angegebenen Begrenzungen einzuhalten.“

Im Teil A wird der Eintrag unter 2 c) wie folgt geändert:

"Schutzkolloide und Verdickungsmittel für die Weiterverarbeitung:

Polyvinylalkohol (Viskosität der 4\%igen wässrigen Lösung bei $20^{\circ} \mathrm{C}$, sofern der Polyvinylalkohol als Schutzkolloid verwendet wird, mindestens $2 \mathrm{cP}$, sofern er als Verdickungsmittel verwendet wird, mindestens $4 \mathrm{cP}$ )

Polymerisate und Copolymerisate aus den unter Nr. 1 genannten Monomeren, wobei die dort angegebenen Beschränkungen einzuhalten sind

Polyacrylsäure, Isopropanol-modifiziert"

In Teil A Nr. 6 wird das Wort „Kunststoff-Dispersionen“ durch „PolymerDispersionen“ ersetzt.

Überschrift und Einleitung von Teil B werden wie folgt geändert:

„Teil B: Beschichtungen auf der Grundlage von Polymer-Dispersionen als Käseüberzüge, die nicht zum Verzehr bestimmt sind.

Bei Einhaltung der nachstehenden Empfehlungen kann davon ausgegangen werden, dass der beim Herstellen, Behandeln und Inverkehrbringen von Käseüberzügen aus Polymer-Dispersionen bestehenden Sorgfaltspflicht entsprochen wird:
Es wird hiermit empfohlen, bei der Herstellung der Polymer-Dispersionen nur folgende Stoffe zu verwenden:“

\section{Polyterephthal- säurediolester}

Stand vom 01.06.2013

Die Empfehlung, zuletzt geändert nach dem Stand vom 01.03.2011 [Bundesgesundheitsblatt - Gesundheitsforschung - Gesundheitsschutz 54 (2011) 666-668], wird wie folgt geändert:

Nr. 4. („Zur Herstellung thermoplastischer Mischkondensate ...") wird gestrichen.

\section{Mischpolymerisate aus Ethylen, Propylen, Butylen, Vinylestern und ungesättigten aliphatischen Säuren sowie deren Salzen und Estern}

Stand vom 01.06.2013

Die Empfehlung, zuletzt geändert nach dem Stand vom 01.01.2010 [Bundesgesundheitsblatt - Gesundheitsforschung - Gesundheitsschutz 53 (2010) 86], wird wie folgt geändert und ergänzt:

Aus Empfehlung XXXV werden folgende Substanzen gestrichen:

Im Abschnitt A unter $2 \mathrm{c}$ (Reste folgender Emulgatoren) wird der Eintrag „Al$\operatorname{kyl}\left(\mathrm{C}_{10}-\mathrm{C}_{20}\right)$ sulfonate " gestrichen. Die dazugehörige Begrenzung „insgesamt höchstens 3,0\%" wird ebenfalls gestrichen.

Nach den restlichen beiden Substanzen („Alkylarylsulfonate“, „Alkylaryloxethylate") wird folgende Formulierung eingefügt: „Teilweise abgedeckt über die Verordnung (EU) Nr. 10/2011. Wo dies der Fall ist, sind die dort angegebenen Beschränkungen einzuhalten. Der Restgehalt der hier aufgeführten Emulgatoren darf $3,0 \%$ nicht überschreiten."

Im Abschnitt A wird die Nummer 3 (Zusatzstoffe) komplett gestrichen.

Im Abschnitt B unter Nummer 3 (Zusatzstoffe) wird der erste Satz geändert in: „Bei der Verarbeitung der Mischpoly- merisate dieses Abschnitts dürfen neben den bereits gemäß Verordnung (EU) Nr. 10/2011 zugelassenen Additiven folgende weitere Stoffe zugesetzt werden:“. Die Nummer 3 c (Verarbeitungshilfen) wird gestrichen.

\section{Papiere, Kartons und Pappen für den Lebensmittelkontakt}

Stand vom 01.06.2013

Die Empfehlung, zuletzt geändert nach dem Stand vom 01.01.2012 [Bundesgesundheitsblatt - Gesundheitsforschung - Gesundheitsschutz 55 (2012) 291-294], wird wie folgt geändert und ergänzt:

Die Vorbemerkungen zur Empfehlung XXXVI werden wie folgt neu gefasst:

1) Die vorliegende Empfehlung gilt für ein- und mehrlagige Bedarfsgegenstände aus Papier, Karton oder Pappe, die bestimmungsgemäß oder vorhersehbar mit Lebensmitteln in Berührung kommen oder auf diese einwirken. Sie schließt Papiere, Kartons und Pappen ein, die dazu bestimmt sind, bei Temperaturen bis $\mathrm{zu} 90^{\circ} \mathrm{C}$ (Warmhalten und Wiedererwärmen von Speisen) eingesetzt zu werden. Für die folgenden, speziellen Anwendungsbereiche (z. B. höhere Temperaturbereiche) ist jedoch die jeweilige Spezial-Empfehlung zu beachten:

- Für Papiere, die bestimmungsgemäß einer Heißextraktion unterworfen werden (z. B. Kochbeutel, Teebeutel, Heißfilterpapiere), und für Filterschichten, die bestimmungsgemäß einer Extraktion (Filtration) unterworfen werden, gilt Empfehlung XXXVI/1.

- Für Papiere, Kartons und Pappen, die beim Backen mit Lebensmitteln in Berührung kommen oder auf diese einwirken, gilt Empfehlung XXXVI/2. Sie gilt auch für Anwendungen in Mikrowellenherden.

- Für Saugeinlagen auf Basis von Cellulosefasern für die Verpackung von Lebensmitteln gilt Empfehlung XXXVI/3. 
2) Bezüglich der Substanzgruppen, die in dieser Empfehlung geregelt werden, ist folgende Abgrenzung zu beachten:

a) Diese Empfehlung regelt Papierrohstoffe (Abschnitt A), Fabrikationshilfsstoffe (Abschnitt B) und spezielle Papierveredelungsstoffe (Abschnitt C), die im Prozess zur Herstellung von Papieren, Kartons und Pappen für den Lebensmittelkontakt eingesetzt werden. Im Papierproduktionsprozess werden darüber hinaus auch Substanzen verwendet, die lediglich der Reinhaltung oder dem Korrosionsschutz der Papiermaschine dienen. Diese Substanzen sind vom Regelungsbereich der BfR-Empfehlungen zu Papier ausgeschlossen. Die diesbezüglichen Pflichten zur Einhaltung der geltenden lebensmittelrechtlichen Vorschriften (insbesondere Artikel 3 der Verordnung (EU) Nr. 1935/2004) liegen beim Hersteller bzw. Inverkehrbringer des Papiers ${ }^{\text {Fußnote }}$. Sind dennoch Substanzen in dieser Empfehlung aufgeführt, die unter die oben genannte Anwendung fallen, so wurden diese vor Einführung dieser Handhabungsweise im Jahr 2013 aufgenommen.

b) Stoffe, die zur Herstellung der im Abschnitt A aufgeführten Papierrohstoffe oder zur Formulierung der in den Abschnitten B und C aufgeführten Wirksubstanzen dienen (wie z. B. Emulgatoren, Lösemittel, Stellmittel, Stabilisatoren, $\mathrm{pH}$ Regulatoren), sind nicht Gegenstand dieser BfR-Empfehlung. Für ihre Verwendung gelten die Anforderungen des Artikels 3 der Verordnung (EU) Nr. 1935/2004 Fußnote. Sind dennoch Substanzen in dieser Empfehlung aufgeführt, die unter die genannten Anwendungen fallen, so wurden diese vor Einführung dieser Handhabungsweise im Jahr 2013 aufgenommen. Konservierungsstoffe, die zum Schutz der Formulierung gegen mikrobiellen Verderb verwendet werden, sowie Schleimverhinderungsmittel blei- ben nach wie vor Bestandteil dieser Empfehlung.

Die Fußnote erhält folgenden Wortlaut: „Zur Orientierung bezüglich der Einhaltung der Verantwortung des Herstellers können u. a. folgende Regelwerke bzw. Bewertungen zu Rate gezogen werden: Andere Empfehlungen des BfR, Bewertungen der Europäischen Behörde für Lebensmittelsicherheit bzw. des Wissenschaftlichen Lebensmittelausschusses (Scientific Committee on Food - SCF), Verordnung (EU) Nr. 10/2011, europäische Regelungen zu Lebensmittelzusatzstoffen oder Trinkwasser. Darüber hinaus kann eine eigenverantwortliche Bewertung durch den Hersteller erfolgen."

3) Methoden für die Untersuchung von Bedarfsgegenständen aus Papier, Karton und Pappe sind unter dem Titel „Untersuchung von Papieren, Kartons und Pappen für den Lebensmittelkontakt" über den Verband Deutscher Papierfabriken e.V. (VDP), Bonn, zu beziehen.

4) Die fertigen Erzeugnisse (Papiere, Kartons und Pappen) dürfen keine konservierende Wirkung auf die mit ihnen in Kontakt kommenden Lebensmittel ausüben.

5) Soweit in dieser Empfehlung der Einsatz bestimmter Stoffe begrenzt wird, beziehen sich die angegebenen Einsatzmengen - wenn nicht die Oberfläche als Bezugsmaßstab angegeben ist - auf das trockene Fertigerzeugnis, soweit nicht anders angegeben.

6) Wird bei der Herstellung eines Papiers, eines Kartons oder einer Pappe die Einsatzmenge eines Fabrikationshilfsstoffs auf Grund seines breiten Wirkungsspektrums an mehreren Stellen der Empfehlung genannt, so gilt als duldbarer Zusatz der höchste der angegebenen Zahlenwerte. Eine Summierung der angegebenen Einsatzmengen ist nicht statthaft.

7) Der Gehalt an Pentachlorphenol im fertigen Papier darf höchstens $0,15 \mathrm{mg} / \mathrm{kg}$ betragen.
8) Bei Gehalten von Metallionen in $\mathrm{Pa}$ pieren (bestimmt im Kaltwasserextrakt) von nicht mehr als A $\mu \mathrm{g} / \mathrm{g}$ Papier, ist davon auszugehen, dass unter praxisnahen Bedingungen auf das Lebensmittel nichts übergeht. Für A gilt: Cadmium 0,5, Blei 3 und Quecksilber 0,3 $\mu \mathrm{g} / \mathrm{g}$ Papier. Die Prüfung entfällt bei der Untersuchung von Papier, Karton und Pappe, die für den Kontakt mit trockenen und nicht fettenden Lebensmitteln bestimmt sind.

9) Azofarbstoffe gem. Anlage 1 ( zu $₫ 3$ ), Nr. 7 der Bedarfsgegenständeverordnung dürfen bei der Herstellung von Papieren, Kartons und Pappen nicht verwendet werden.

10) Besteht bei mehrlagigen, mehrschichtigen oder beschichteten Bedarfsgegenständen die mit den Lebensmitteln in Berührung kommende Lage oder Schicht aus Papier, Karton oder Pappe, muss sie dieser Empfehlung entsprechen. Auch von den anderen Lagen oder Schichten dürfen keine Stoffe auf die Lebensmittel oder deren Oberfläche übergehen, ausgenommen gesundheitlich, geruchlich und geschmacklich unbedenkliche Anteile.

Gegen die Verwendung von Papieren, Kartons und Pappen als Bedarfsgegenstände im Sinne von $₫ 2$ Abs. 6 Nr. 1 des Lebensmittel- und Futtermittelgesetzbuches (LFGB) bestehen keine Bedenken, sofern die Bedarfsgegenstände sich für den vorgesehenen Zweck eignen und folgende weitere Voraussetzungen erfüllt sind:“

Der Abschnitt A. II. (Rohstoffadditive) wird gestrichen. Dafür wird die Überschrift des Abschnittes A mit folgender Fußnote ergänzt: „Rohstoffadditive gehören seit dem Jahr 2013 nicht mehr zum Geltungsbereich dieser Empfehlung (siehe Vorbemerkungen, Nummer 2). Folgende Substanzen sind vor Einführung dieser Regelung bewertet und in diese Empfehlung aufgenommen worden: 
a) Xylanase (In den Fertigerzeugnissen darf keine Restaktivität des Enzyms nachweisbar sein.)

b) Diethylentriamin-pentamethylenphosphonsäure, höchstens $0,22 \%$, bezogen auf den trockenen Faserstoff

c) Kaliumsulfit, höchstens 0,01\%

d) Iminodibernsteinsäure, Di-Natriumsalz, höchstens $0,17 \%$, bezogen auf den trockenen Faserstoff

e) Aktivkohle (Die Aktivkohle muss den Reinheitsanforderungen für E 153 gemäß der Verordnung (EU) 231/2012 entsprechen.)

Der Einsatz der zuvor genannten Substanzen unter Beachtung der oben genannten Einschränkungen entspricht weiterhin dieser Empfehlung.“

Die Verwerndung von Anthrachinon als Rohstoffadditiv wird nicht weiter empfohlen.

Fußnote 14, welche sich auf den Eintrag unter B. III. (Retentionsmittel) Nr. 1 bezieht, wird wie folgt geändert: „Als Formulierungshilfsmittel können Kohlenwasserstoff-Lösemittel (paraffinisch, naphthenisch, mit einer Kohlenstoffzahl von $\mathrm{C}_{10}-\mathrm{C}_{20}$ ) verwendet werden, die den Reinheitsanforderungen für flüssige Paraffine (s. 155. Mitt., Bundesgesundheitsbl. 25 (1982) 192) entsprechen. Der Übergang der Anteile mit einer Kohlenstoffzahl von $\mathrm{C}_{10}-\mathrm{C}_{16}$ auf Lebensmittel darf den vorübergehend festgesetzten Wert von $12 \mathrm{mg} / \mathrm{kg}$ nicht überschreiten. Für Anteile mit einer Kohlenstoffzahl von $\mathrm{C}_{16}-\mathrm{C}_{20}$ darf der Übergang auf Lebensmittel den vorübergehend festgesetzten Wert von $4 \mathrm{mg} / \mathrm{kg}$ nicht überschreiten.“

Unter B. V. (Dispergier- und Flotationsmittel) wird folgender Eintrag ergänzt: „Ester von Polyethylenglycol mit Rizinusöl, höchstens $5 \mathrm{mg} / \mathrm{dm}^{2 “}$

Unter B. VI. (Schaumverhütungsmittel) werden folgende Einträge ergänzt:

„Polypropylenglycol, höchstens $1 \mathrm{mg} / \mathrm{dm}^{2}$ Poly(ethylenpropylen)glycol, höchstens $1 \mathrm{mg} / \mathrm{dm}^{2 *}$

Weiterhin werden hier folgende drei Substanzen ergänzt: „a) 2,4,7,9-Tetramethyl-5-decin-4,7-diol

b) 3,6-Dimethyl-4-octin-3,6-diol

c) 2,5,8,11-Tetramethyl-6-dodecin5,8-diol“

Für diese drei Substanzen wird folgende Begrenzung eingefügt: „Der Übergang dieser drei Substanzen aufs Lebensmittel darf in der Summe 0,05 mg/kg Lebensmittel nicht überschreiten.“

Im Abschnitt C IV. (Mittel zur Oberflächenveredelung und -beschichtung) wird der Eintrag unter der Nr. 20 („Mischung aus Bis-(diethanolammonium)mono-1H-1H, 2H-2H-perfluoralkylortho-phosphat und Diethanolammoniumbis- $(1 \mathrm{H}-1 \mathrm{H}, 2 \mathrm{H}-2 \mathrm{H}$-perfluoralkyl)orthophosphat") einschließlich der dazugehörigen Beschränkungen gestrichen.

\section{XXXVI/1 Koch- und Heißfilterpapiere und Filterschichten}

Stand: 01.06.2013

Die Empfehlung, zuletzt geändert nach dem Stand vom 01.01.2012 [Bundesgesundheitsblatt - Gesundheitsforschung - Gesundheitsschutz 55 (2012) 291-294], wird wie folgt geändert und ergänzt: $\mathrm{Zu}$ Beginn der Empfehlung wird folgender Text eingefügt:

„Vorbemerkungen

Diese Empfehlung regelt allgemeine Rohstoffe (Abschnitt I), allgemeine Fabrikationshilfsstoffe (Abschnitt II) und spezielle Rohstoffe und Fabrikationshilfsstoffe (Abschnitt III), die im Prozess zur Herstellung von Papieren, Kartons und Pappen für den Lebensmittelkontakt eingesetzt werden. Im Papierproduktionsprozess werden darüber hinaus auch Substanzen verwendet, die lediglich der Reinhaltung oder dem Korrosionsschutz der Papiermaschine dienen. Diese Substanzen sind vom Regelungsbereich der BfR-Empfehlungen zu Papier ausgeschlossen. Die diesbezüglichen Pflichten zur Einhaltung der geltenden lebensmittelrechtlichen Vorschriften (insbesondere Artikel 3 der Verordnung (EU) Nr. 1935/2004) liegen beim
Hersteller bzw. Inverkehrbringer des $\mathrm{Pa}$ piers $^{\text {Fußnote }}$. Sind dennoch Substanzen in dieser Empfehlung aufgeführt, die unter die oben genannte Anwendung fallen, so wurden diese vor Einführung dieser Handhabungsweise im Jahr 2013 aufgenommen.

Stoffe, die zur Herstellung der im Abschnitt I aufgeführten Papierrohstoffe oder zur Formulierung der in den Abschnitten II und III aufgeführten Wirksubstanzen dienen (wie z. B. Emulgatoren, Lösemittel, Stellmittel, Stabilisatoren, $\mathrm{pH}$-Regulatoren), sind nicht Gegenstand dieser BfR-Empfehlung. Für ihre Verwendung gelten die Anforderungen des Artikels 3 der Verordnung (EU)

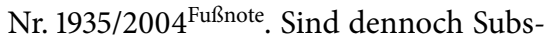
tanzen in dieser Empfehlung aufgeführt, die unter die genannten Anwendungen fallen, so wurden diese vor Einführung dieser Handhabungsweise im Jahr 2013 aufgenommen. Konservierungsstoffe, die zum Schutz der Formulierung gegen mikrobiellen Verderb verwendet werden, sowie Schleimverhinderungsmittel bleiben nach wie vor Bestandteil dieser Empfehlung.“

Die Fußnote erhält folgenden Wortlaut: „Zur Orientierung bezüglich der Einhaltung der Verantwortung des Herstellers können u. a. folgende Regelwerke bzw. Bewertungen zu Rate gezogen werden: Andere Empfehlungen des BfR, Bewertungen der Europäischen Behörde für Lebensmittelsicherheit bzw. des Wissenschaftlichen Lebensmittelausschusses (Scientific Committee on Food - SCF), Verordnung (EU) Nr. 10/2011, europäische Regelungen zu Lebensmittelzusatzstoffen oder Trinkwasser. Darüber hinaus kann eine eigenverantwortliche $\mathrm{Be}$ wertung durch den Hersteller erfolgen."

Unter II. F. (Schaumverhütungsmittel) werden folgende drei Substanzen ergänzt:

„a) 2,4,7,9-Tetramethyl-5-decin-4,7-diol

b) 3,6-Dimethyl-4-octin-3,6-diol

c) 2,5,8,11-Tetramethyl-6-dodecin-5,8diol“"

Für diese drei Substanzen wird folgende Begrenzung eingefügt: „Der Übergang dieser drei Substanzen aufs Lebensmittel 
darf in der Summe 0,05 mg/kg Lebensmittel nicht überschreiten.“

\section{XXXVI/2 Papiere, Kartons und Pappen für Backzwecke}

Stand: 01.06.2013

Die Empfehlung, zuletzt geändert nach dem Stand vom 01.02.2013 [Bundesgesundheitsblatt - Gesundheitsforschung - Gesundheitsschutz 55 (2012) 291-294], wird wie folgt geändert und ergänzt: In den Vorbemerkungen der Empfehlung wird die Nummer 1 wie folgt ergänzt: „Diese Empfehlung regelt Papierrohstoffe (Abschnitt I), Fabrikationshilfsstoffe (Abschnitt II) und spezielle Papierveredelungsstoffe (Abschnitt III), die im Prozess zur Herstellung von Papieren, Kartons und Pappen für den Lebensmittelkontakt eingesetzt werden, sowie Fertigerzeugnisse (Abschnitt IV). Im Papierproduktionsprozess werden darüber hinaus auch Substanzen verwendet, die lediglich der Reinhaltung oder dem Korrosionsschutz der Papiermaschine dienen. Diese Substanzen sind vom Regelungsbereich der BfR-Empfehlungen zu Papier ausgeschlossen. Die diesbezüglichen Pflichten zur Einhaltung der geltenden lebensmittelrechtlichen Vorschriften (insbesondere Artikel 3 der Verordnung (EU) Nr. 1935/2004) liegen beim Hersteller bzw. Inverkehrbringer des Papiers ${ }^{\text {Fußnote }}$. Sind dennoch Substanzen in dieser Empfehlung aufgeführt, die unter die oben genannte Anwendung fallen, so wurden diese vor Einführung dieser Handhabungsweise im Jahr 2013 aufgenommen.

Stoffe, die zur Herstellung der im Abschnitt I aufgeführten Papierrohstoffe oder zur Formulierung der in den Abschnitten II und III aufgeführten Wirksubstanzen dienen (wie z. B. Emulgatoren, Lösemittel, Stellmittel, Stabilisatoren, $\mathrm{pH}$-Regulatoren), sind nicht Gegenstand dieser BfR-Empfehlung. Für ihre Verwendung gelten die Anforderungen des Artikels 3 der Verordnung (EU) Nr. 1935/2004 $4^{\text {Fußnote }}$. Sind dennoch Substanzen in dieser Empfehlung aufgeführt, die unter die genannten Anwendungen fallen, so wurden diese vor Einführung dieser Handhabungsweise im Jahr 2013 aufgenommen. Konservierungsstoffe, die zum Schutz der Formulierung gegen mikrobiellen Verderb verwendet werden, sowie Schleimverhinderungsmittel bleiben nach wie vor Bestandteil dieser Empfehlung."

Die Fußnote erhält folgenden Wortlaut: „Zur Orientierung bezüglich der Einhaltung der Verantwortung des Herstellers können u. a. folgende Regelwerke bzw. Bewertungen zu Rate gezogen werden: Andere Empfehlungen des BfR, Bewertungen der Europäischen Behörde für Lebensmittelsicherheit bzw. des Wissenschaftlichen Lebensmittelausschusses (Scientific Committee on Food - SCF), Verordnung (EU) Nr. 10/2011, europäische Regelungen zu Lebensmittelzusatzstoffen oder Trinkwasser. Darüber hinaus kann eine eigenverantwortliche Bewertung durch den Hersteller erfolgen."

Der Abschnitt I B (Rohstoffadditiv) wird gestrichen.

Fußnote 13 wird am Ende wie folgt ergänzt: „2-Methyl-4-isothiazolin-3-on: $80 \mu \mathrm{g} / \mathrm{dm}^{2 *}$

Der Abschnitt unter der Nummer III C (Farbmittel und optische Aufheller) wird wie folgt neu gefasst:

„Für Backpapiere gemäß dieser Empfehlung dürfen nur die im Folgenden aufgeführten Farbmittel und optischen Aufheller verwendet werden:

a) Farbmittel

\section{Eisen-III-oxid}

2. Darüber hinaus dürfen bei mehrlagigen oder mehrschichtigen Papieren, Kartons und Pappen in der nicht dem Lebensmittel zugewandten Lage oder Schicht Farbmittel zur Weißnuancierung eingesetzt werden, sofern sichergestellt ist, dass diese bei bestimmungsgemäßem Gebrauch der Bedarfsgegenstände nicht auf Lebensmittel übergehen ${ }^{3}$

b) Optische Aufheller

(wurden bislang nicht beantragt)“
Fußnote 14 wird wie folgt neu gefasst: „Farbmittel zum Einfärben für die in diesem Abschnitt aufgeführten Oberflächenveredelungsmittel dürfen nicht verwendet werden."

Unter der Nummer III D (Mittel zur Oberflächenveredelung der dem Füllgut anliegenden Seite) wird die Eintragung Nummer 9 ("Copolymer aus Perfluoralkylethylacrylat, Vinylacetat und N,NDimethylaminoethylmethacrylat, höchstens $0,6 \%$ ") gestrichen.

\section{XXXVI/3 Saugeinlagen auf Basis von Cellulosefasern für die Verpackung von Lebensmitteln}

\section{Stand: 01.06.2013}

Die Empfehlung, zuletzt geändert nach dem Stand vom 01.01.2012 [Bundesgesundheitsblatt - Gesundheitsforschung - Gesundheitsschutz 55 (2012) 291-294], wird wie folgt geändert und ergänzt: Am Anfang der Empfehlung wird folgender Text eingefügt:

„Vorbemerkungen

Diese Empfehlung regelt Faserstoffe (Abschnitt I A) und Fabrikationshilfsstoffe (Abschnitt I B), die im Prozess zur Herstellung von Papieren, Kartons und Pappen für den Lebensmittelkontakt eingesetzt werden, sowie Fertigerzeugnisse (Abschnitt III). Im Papierproduktionsprozess werden darüber hinaus auch Substanzen verwendet, die lediglich der Reinhaltung oder dem Korrosionsschutz der Papiermaschine dienen. Diese Substanzen sind vom Regelungsbereich der BfR-Empfehlungen zu Papier ausgeschlossen. Die diesbezüglichen Pflichten zur Einhaltung der geltenden lebensmittelrechtlichen Vorschriften (insbesondere Artikel 3 der Verordnung (EU) Nr. 1935/2004) liegen beim Hersteller bzw. Inverkehrbringer des Pa-

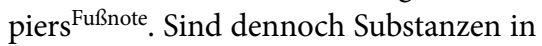
dieser Empfehlung aufgeführt, die unter die oben genannte Anwendung fallen, so wurden diese vor Einführung dieser Handhabungsweise im Jahr 2013 aufgenommen. 
Stoffe, die zur Herstellung der im Abschnitt I A aufgeführten Faserstoffe oder zur Formulierung der im Abschnitt I B aufgeführten Wirksubstanzen dienen (wie z. B. Emulgatoren, Lösemittel, Stellmittel, Stabilisatoren, $\mathrm{pH}$-Regulatoren), sind nicht Gegenstand dieser BfR-Empfehlung. Für ihre Verwendung gelten die Anforderungen des Artikels 3 der Verordnung (EU) Nr. 1935/2004 Fußnote. Sind dennoch Substanzen in dieser Empfehlung aufgeführt, die unter die genannten Anwendungen fallen, so wurden diese vor Einführung dieser Handhabungsweise im Jahr 2013 aufgenommen. Konservierungsstoffe, die zum Schutz der Formulierung gegen mikrobiellen Verderb verwendet werden, sowie Schleimverhinderungsmittel bleiben nach wie vor Bestandteil dieser Empfehlung.“

Die Fußnote erhält folgenden Wortlaut: „Zur Orientierung bezüglich der Einhaltung der Verantwortung des Herstellers können u. a. folgende Regelwerke bzw. Bewertungen zu Rate gezogen werden: Andere Empfehlungen des BfR, Bewertungen der Europäischen Behörde für Lebensmittelsicherheit bzw. des Wissenschaftlichen Lebensmittelausschusses (Scientific Committee on Food - SCF), Verordnung (EU) Nr. 10/2011, europäische Regelungen zu Lebensmittelzusatzstoffen oder Trinkwasser. Darüber hinaus kann eine eigenverantwortliche Bewertung durch den Hersteller erfolgen."

Unter I B Nummer 6 (Schaumverhütungsmittel) werden folgende drei Substanzen ergänzt:
,a) 2,4,7,9-Tetramethyl-5-decin-4,7-diol
b) 3,6-Dimethyl-4-octin-3,6-diol
c) 2,5,8,11-Tetramethyl-6-dodecin-5,8- diol"

Für diese drei Substanzen wird folgende Begrenzung eingefügt: „Der Übergang dieser drei Substanzen aufs Lebensmittel darf in der Summe 0,05 mg/kg Lebensmittel nicht überschreiten.“

\section{Temperaturbeständige Beschichtungssysteme aus Polymeren für Brat-, Koch- und Backgeräte}

Stand: 01.06.2013

Die Empfehlung, zuletzt geändert nach dem Stand vom 01.03.2011 [Bundesgesundheitsblatt - Gesundheitsforschung - Gesundheitsschutz 54 (2011) 666-668], wird wie folgt geändert und ergänzt: Unter 2.1.4.1 (Emulgatoren) wird folgender Eintrag ergänzt: „Perfluor[(2-ethyloxy-ethoxy)essigsäure], maximal 0,83\%, bezogen auf das Polymer. Die Substanz darf nur in Fluorpolymeren eingesetzt werden, die mindestens 10 min lang bei $>300^{\circ} \mathrm{C}$ gesintert werden. $"$ 